\title{
An Investigation of the Skin Barrier Restoring Effects of a Cream and Lotion Containing Ceramides in a Multi-vesicular Emulsion in People with Dry, Eczema-Prone, Skin: The RESTORE Study Phase 1
}

\author{
Simon G. Danby (D) - Paul V. Andrew - Kirsty Brown • \\ John Chittock · Linda J. Kay · Michael J. Cork (D)
}

Received: June 12, 2020 / Published online: July 15, 2020

(C) The Author(s) 2020

\begin{abstract}
Introduction: The replenishment of skin lipids depleted in the dry skin state is a desirable therapeutic target to restore skin moisturization; however, there is limited evidence demonstrating the success of this approach through the use of topical emollients. The purpose of this study was to provide evidence of the benefits of a cream and equivalent lotion containing skin lipids in a multi-vesicular emulsion for the management of dry skin. The hypothesis was that the test cream and test lotion could sustain skin moisturization for longer than
\end{abstract}

Digital Features To view digital features for this article go to https://doi.org/10.6084/m9.figshare.12620618.

S. G. Danby $(\varangle) \cdot$ P. V. Andrew · K. Brown .

J. Chittock · L. J. Kay · M. J. Cork

Sheffield Dermatology Research, Department of

Infection, Immunity and Cardiovascular Disease,

The University of Sheffield Medical School,

Sheffield, UK

e-mail: s.danby@sheffield.ac.uk

M. J. Cork

Sheffield Teaching Hospitals NHS Foundation Trust, The Royal Hallamshire Hospital, Sheffield, UK

\section{J. Cork}

Sheffield Children's NHS Foundation Trust,

Sheffield Children's Hospital, Western Bank,

Sheffield, UK

e-mail:m.j.cork@sheffield.ac.uk traditional emollients by sustainably delivering skin lipids.

Methods: A double-blind intra-subject vehiclecontrolled single open-application test on the lower legs in people with dry, atopic dermatitis (atopic eczema)-prone, skin was conducted. There were six treatment sites, three per lower leg in each participant, which were treated with the test cream, the test lotion, three reference creams commonly prescribed in the UK and no treatment as a control. After baseline measurements of skin hydration, $100 \mu \mathrm{l}$ of the test/reference creams was applied to each of the relevant treatment sites (random site allocation). Following treatment, measurements of skin hydration and scoring of visual dryness was conducted at timed intervals $(3,6,12$ and $24 \mathrm{~h}$ post-product application).

Results: The test cream and lotion both significantly increased skin hydration and reduced skin dryness for at least $24 \mathrm{~h}$ following a single application compared to a no treatment control site. Compared to three reference emollient creams the test cream and test lotion were the only products capable of sustaining clinically meaningful improvements in skin moisturization for $24 \mathrm{~h}$.

Conclusion: The sustained moisturization imparted by the test products reduces the need for frequent emollient application, often requiring 3-4 applications per day for traditional emollients, and should reduce the high burden of managing dry skin conditions like 
atopic dermatitis.Keywords: Atopic dermatitis; Capacitance; Ceramide; Dryness; Emollient; Hydration; Moisturizer; Xerosis

\section{Key Summary Points}

\section{Why carry out this study?}

Stratum corneum lipid replacement therapy is a promising strategy for treating dry skin conditions like atopic dermatitis.

Dry skin is a common condition, affecting around $20 \%$ of children and $30-75 \%$ of adults over the age of 60 .

The aim was to provide evidence of the benefits of a cream and equivalent lotion containing skin lipids in a multi-vesicular emulsion for the management of dry skin.

\section{What was learned from the study?}

In contrast to traditional emollients currently prescribed for dry skin, which require regular application 3-4 times per day, the test products imparted sustained moisturization lasting for more than $24 \mathrm{~h}$.

Reducing the number of applications required to keep the skin hydrated will help lessen the high burden of managing dry skin conditions.

\section{INTRODUCTION}

Ceramides, cholesterol and free fatty acids are important constituents of the stratum corneum (SC), the outermost layer of the skin. Filling the space between the corneocytes they form a highly ordered matrix, referred to as the lipid lamellae, that forms an effective waterproof barrier. The composition and structure of the lipid lamellae is critically important to the permeability barrier function of the skin. Low SC ceramide levels are associated with xerosis (skin dryness) and reduced skin barrier function
$[1,2]$. In the dry skin condition atopic dermatitis/eczema (AD) there is a reduction in the amount of total SC lipids owing to a significant ceramide deficiency in both lesional and nonlesional skin [3]. SC lipid insufficiency in healthy infants is linked to decreased stratum corneum hydration (SCH) [4]. SC lipid levels are also reduced in people over the age of 60 compared to young adults, and are associated with the increased incidence of skin dryness in this population [5-7]. Skin supplementation with skin lipids, including ceramides, is therefore a potentially important mechanism for supporting the skin's barrier function and maintaining normal $\mathrm{SCH}$ across the ages in the general population [8].

Here we present the findings of the first study in a program of work to establish the effects of a ceramide-containing cream and lotion on the skin, henceforth referred to as the test cream (TC) and test lotion (TL). Both test products contain a range of skin lipids, including ceramides 1, 3 and 6-II, triglycerides (source of fatty acids) and cholesterol, important for the formation of the skin barrier. Together with the humectant glycerol these active moisturizing agents are delivered using a multi-vesicular emulsion (MVE) controlled-release system. Unlike traditional oil-in-water emulsions with oil droplets dispersed in a continuous water phase, MVE is comprised of multilayer concentric spheres of emulsified oil and water suspended in an aqueous medium. The moisturizers within each layer are released sequentially thereby prolonging the hydrating effects of the MVE moisturizer compared to traditional moisturizers $[9,10]$. The National Institute for Health and Care Excellence (NICE) guidelines on the treatment of $\mathrm{AD}$ suggest applying moisturizers four times per day, which can be an onerous task. The MVE technology in the test products is purported to deliver sustainable hydration over an extended period and so reduce the number of applications required per day. The aim of this study was to determine the duration of $\mathrm{SCH}$ imparted by the test products and compare it with current 'traditional' moisturizers marketed for the treatment of dry skin in the UK. The most common type of emollient cream prescribed by the National 
Health Service (NHS) is the simple emollient cream based upon paraffins (accounting for $37 \%$ of all prescriptions in 2018). Of this class of emollient, we selected three reference products that together represented $22.1 \%$ of emollient cream prescriptions in this period.

\section{METHODS}

This study was conducted at the Sheffield Dermatology Research, Skin Barrier Research Facility from May 2018 until June 2018. Recruitment took place during April 2018. The University of Sheffield Research Ethics Committee (UREC) approved the study, under the project reference 018584 . The study was performed in accordance with the Helsinki Declaration of 1964, and its later amendments, and all subjects provided informed consent to participate.

\section{Study Design}

A double-blind intra-subject vehicle-controlled single open-application test on the lower legs in people with dry eczema-prone skin was conducted. The lower legs were chosen because of their increased propensity for skin dryness, and were divided into six test sites $(4 \times 5 \mathrm{~cm}$ each $)$ on the lateral side of the tibia ridge. Each test site received a single $100-\mu \mathrm{l}$ application of either the TC, the TL, the reference creams (vehicle controls, see Table 1 for details) or no treatment (no treatment control, NTC). Randomized site allocation was utilized to minimize site-dependent effects using a randomization list generated at www.randomisation.com. Product identities were concealed from the investigator and participant by assigning each a letter code from A to F. The biophysical properties of the test sites were determined before and at set time points after treatment application. Participants were asked to refrain from washing the test sites until completion of the study.

\section{Participants}

A single cohort of volunteers with dry skin and a predisposition to $\mathrm{AD}$ was recruited.
Recruitment was open to male and female volunteers, who were recruited on a first come first served basis, assuming they met the specified criteria. Inclusion criteria included having a self-reported history of AD defined by the UK Working Party diagnostic criteria, having visibly dry skin on the lower leg, SCH within the test area measuring $\leq 35$ AU and being aged 18 years and over. Exclusion criteria included currently undergoing, or requiring, active drug treatment for $\mathrm{AD}$; a known allergy/hypersensitivity to any of the excipients of the trial preparations; use of other topical products and/ or abrasive cleansers/treatments on the test sites for 1 week prior to and throughout the study; having perceptible differences between the visual dryness scores of the test sites; dermatitis, acne, suntan, hyperpigmentation, multiple nevi, tattoos, blemishes or dense body hair in the test areas; a condition that in the opinion of the investigator contradicts participation in the study; use of any medication that could interfere with the trial aim (e.g. corticosteroids, calcineurin inhibitors, methotrexate) 3 months prior to the start of the study treatment and throughout the study; current participation in an interventional clinical trial. Informed consent was obtained from each participant. All participants received remuneration appropriate for their involvement.

\section{Objective Measurement of SCH}

SCH was measured indirectly via the capacitance method using a Corneometer CM825 (CK electronic GmbH, Cologne, Germany). The corneometer measures in arbitrary units (AU) from 0 to 120; a reading of 0 is recorded when in contact with a surface containing no water and 120 when in direct contact with water. Normal skin typically displays $\mathrm{SCH}$ readings in the region of $35 \mathrm{AU}$, with hydrated skin being above 40 and dry skin below 30. Baseline measurements were taken before product application and at 3, 6, 12 and $24 \mathrm{~h}$ following product application. All assessments were performed in a room maintained at $21 \pm 2{ }^{\circ} \mathrm{C}$ and $38-50 \%$ relative humidity. All test sites were 
Table 1 Investigational products

\begin{tabular}{|c|c|c|}
\hline Name (brand name) & Manufacturer & Ingredients (active moisturizing agents) \\
\hline $\begin{array}{l}\text { Test cream, TC (CeraVe } \\
\text { cream) }\end{array}$ & $\begin{array}{l}\text { L'OREAL, Active } \\
\text { Cosmetics Division }\end{array}$ & $\begin{array}{l}\text { Aqua/water, glycerin, cetearyl alcohol, caprylic/capric triglyceride, cetyl } \\
\text { alcohol, ceteareth-20, petrolatum, dimethicone, phenoxyethanol, } \\
\text { behentrimonium methosulfate, potassium phosphate, } \\
\text { ethylhexylglycerin, sodium lauroyl lactylate, disodium EDTA, } \\
\text { dipotassium phosphate, ceramide NP, ceramide AP, } \\
\text { phytosphingosine, cholesterol, xanthan gum, carbomer, sodium } \\
\text { hyaluronate, tocopherol, ceramide EOP }\end{array}$ \\
\hline $\begin{array}{l}\text { Test lotion, TL (CeraVe } \\
\text { lotion) }\end{array}$ & $\begin{array}{l}\text { L'OREAL, Active } \\
\text { Cosmetics Division }\end{array}$ & $\begin{array}{l}\text { Aqua/water, glycerin, caprylic/capric triglyceride, cetearyl alcohol, cetyl } \\
\text { alcohol, dimethicone, phenoxyethanol, polysorbate 20, ceteareth-20, } \\
\text { behentrimonium methosulfate, polyglyceryl-3 diisostearate, sodium } \\
\text { lauroyl lactylate, ethylhexylglycerin, potassium phosphate, disodium } \\
\text { EDTA, dipotassium phosphate, ceramide NP, ceramide AP, } \\
\text { phytosphingosine, cholesterol, xanthan gum, carbomer, sodium } \\
\text { hyaluronate, tocopherol, ceramide EOP }\end{array}$ \\
\hline $\begin{array}{l}\text { Reference cream 1, RC1 } \\
\text { (Zerobase cream) }\end{array}$ & Thornton \& Ross Ltd & $\begin{array}{l}\text { Purified water, liquid paraffin, white soft paraffin, cetostearyl alcohol, } \\
\text { cetomacrogol, sodium dihydrogen phosphate, chlorocresol, } \\
\text { phosphoric acid }\end{array}$ \\
\hline $\begin{array}{l}\text { Reference cream 2, RC2 } \\
\text { (Epimax cream) }\end{array}$ & Dermato-Logical & $\begin{array}{l}\text { Purified water, white soft paraffin, liquid paraffin, polysorbate } 60 \text {, } \\
\text { cetosteryl alcohol, phenoxyethanol }\end{array}$ \\
\hline $\begin{array}{l}\text { Reference cream 3, RC3 } \\
\text { (Aquamax cream) }\end{array}$ & $\begin{array}{l}\text { Intapharm } \\
\text { Laboratories }\end{array}$ & $\begin{array}{l}\text { Purified water, white soft paraffin, cetostearyl alcohol, liquid paraffin, } \\
\text { polysorbate } 60 \text {, phenoxyethanol }\end{array}$ \\
\hline
\end{tabular}

acclimatised to room conditions for $20 \mathrm{~min}$ before each assessment.

\section{Visual Scoring of Dryness}

Visual dryness was scored by two experienced graders independently using the overall dry skin score (ODS) for dryness where 0 is no dryness and 4 is marked dryness (skin dominated by large scales, advanced roughness, redness present, eczematous changes and cracks). Scoring was conducted before product application and at $3,6,12$ and $24 \mathrm{~h}$ following product application.

\section{Statistical Analysis}

All data were analysed using Prism 7 (GraphPad Software, La Jolla, USA). The primary outcome was the difference in the change in objective
SCH 3, 6, 12 and $24 \mathrm{~h}$ after application between treated sites and the NTC. The secondary outcome was the difference in the change in visual skin dryness 3, 6, 12 and $24 \mathrm{~h}$ after application between treated sites and the NTC. The significance threshold was $p<0.05$. Results are presented as mean \pm standard error of the mean (SEM) unless otherwise indicated. SCH data was significance tested at each time point using a repeated-measures one-way analysis of variance (ANOVA) with a Dunnett's post-test. The study was powered (80\%) to detect clinically relevant differences in SCH of 5 units (sample size $\geq 18$ ), which represents the shift in hydration observed between inactive and active AD [11]. The secondary outcome of skin dryness score was significance tested at each time point using a Friedman test with Dunn's post-test. 
Table 2 Cohort demographics

\begin{tabular}{lll}
\hline Demographic & Full analysis set (FAS) & Per protocol set (PPS) \\
\hline$n$ & 22 & 18 \\
Age (mean) & $41 \pm 2.96$ & $43 \pm 3.33$ \\
Sex & & 7 \\
Male & 10 & 11 \\
Female & 12 & \\
Ethnicity & 19 & 15 \\
White & 2 & 2 \\
South Asian & 1 & 1 \\
Mixed & $2($ range 1-5) & 3 (range 1-5) \\
Fitzpatrick skin type (median) & & \\
Atopic dermatitis (AD) & $5 / 22(23 \%)$ & $5 / 18(28 \%)$ \\
Previously suffered from eczema but it is fully resolved & $8 / 22(36 \%)$ & $6 / 18(33 \%)$ \\
Currently suffer from eczema but my skin is currently clear & $9 / 22(41 \%)$ & $7 / 18(39 \%)$ \\
Currently suffer from eczema and it is currently active/visible & $25.76 \pm 1.81$ & $23.31 \pm 1.55$ \\
SCH & $1.59($ range $0-3)$ & $1.83($ range $1-3)$ \\
Dryness &
\end{tabular}

\section{RESULTS}

A total of 26 volunteers consented to take part in the study, 22 of which completed the study. Of the remaining four participants, three were screening failures and one was unavailable on the study days. Four participants who successfully completed the trial had scores for visual dryness and/or SCH at the baseline visit (which was conducted on a separate occasion to the screening visit) outside the criteria defined in the protocol. Results are therefore presented for all 22 participants who completed the trial (full analysis set, FAS) and for the 18 participants who completed the trial according to the protocol (per protocol set, PPS). There were no other protocol deviations and no missing values.

The demographic characteristics of the study cohort are presented in Table 2 . All 22 participants had a self-reported history of $\mathrm{AD}$, but were not currently undergoing active drug (i.e. antiinflammatory agent) treatment. As per protocol the participants refrained from using emollient products on the test sites for 1 week prior to the first study visit and refrained from washing the test sites from $12 \mathrm{~h}$ before the start of the test until after the final measurements were taken. No adverse events occurred during the conduct of the study.

The effect of a single application of each treatment on SCH and visual dryness over time is presented in Figs. 1 and 2. The overall trend is consistent between both analysis sets, with the exception that the PPS displays a drier, less hydrated, skin condition at baseline on average. The subsequent analysis is presented for the PPS only (and results were consistent with the FAS).

TC and TL were the only treatments found to sustainably increase $\mathrm{SCH}$ by more than $5 \mathrm{AU}$ compared to the NTC (Fig. 1). The greatest change in $\mathrm{SCH}$ was recorded $3 \mathrm{~h}$ after product 
(a)

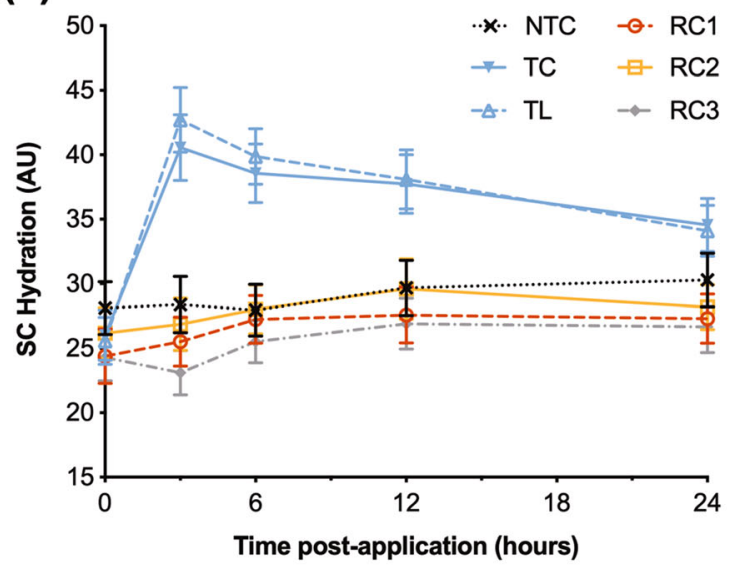

(c)

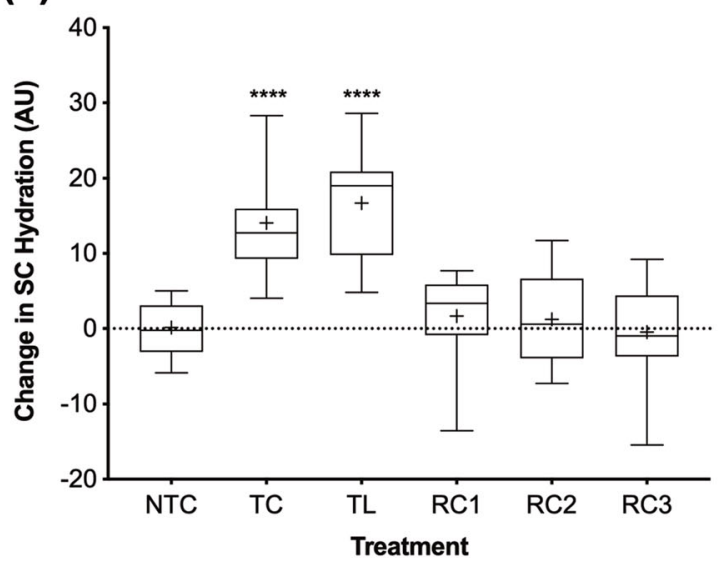

(e)

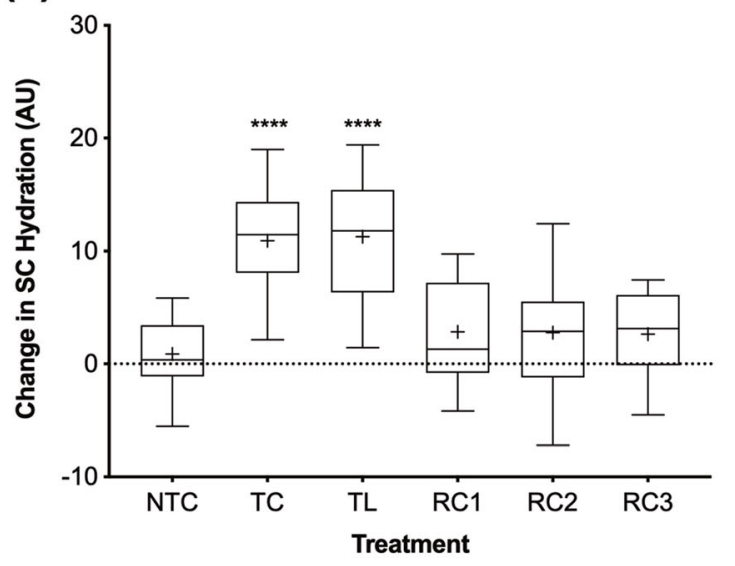

(b)

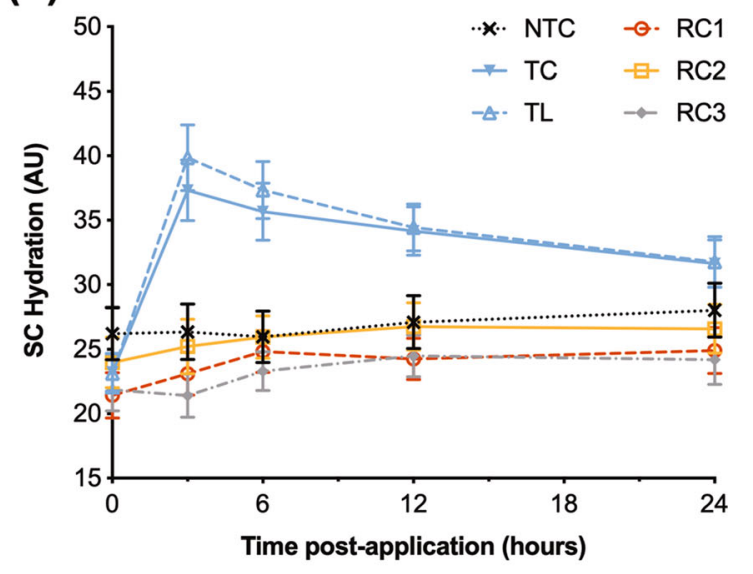

(d)

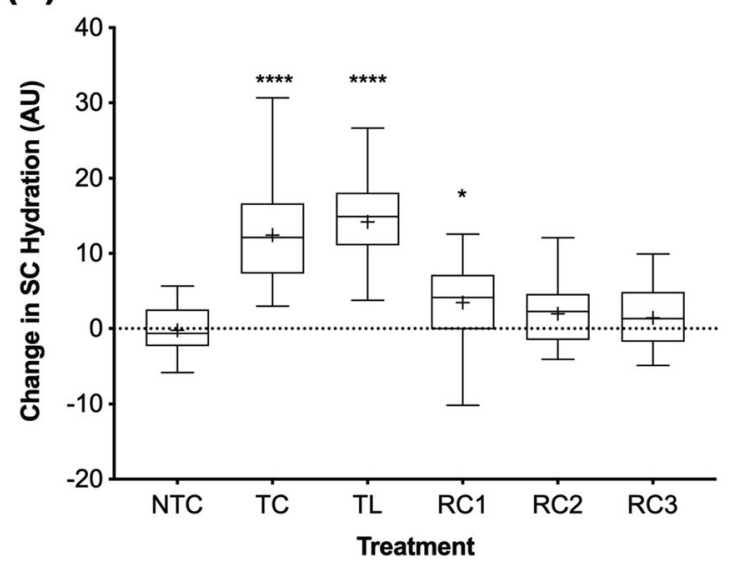

(f)

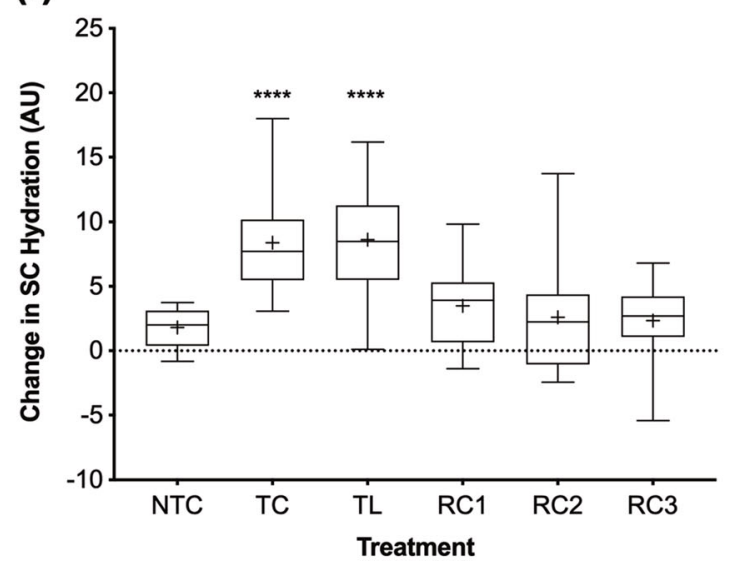


4Fig. 1 Effect of the 6 treatment conditions on SCH (primary analysis). Raw SCH over $24 \mathrm{~h}$ following a single application is displayed for the FAS (a) and PPS (b). Change in SCH from baseline is displayed for the PPS at 3- (c), 6- (d), 12- (e) and 24-h (f) post-treatment. Baseline measurements $(0 \mathrm{~h})$ were taken immediately prior to application of the investigational products. NTC, no treatment control; TC, test cream; TL, test lotion; RC, reference cream. Error bars ( $\mathbf{a}$ and $\mathbf{b}$ ) indicate SEM. Boxes (c-f) indicate the median, 25th and 75th percentiles, with ' + ' for the mean and whiskers indicating the minimum and maximum. Asterisks indicate the results of a Dunnett's post-test comparing each treatment to the NTC $\left({ }^{*} p<0.05,{ }^{* *} p<0.01,{ }^{* * *} p<0.001,{ }^{* * * *} p<0.0001\right)$

application with a mean increase from the NTC of $13.95 \pm 1.58 \mathrm{AU}$ for the TC and $16.59 \pm 1.98 \mathrm{AU}$ for the TL. SCH remained elevated, albeit to a decreasing extent, for $24 \mathrm{~h}$, whereupon the increase in SCH was $6.58 \pm 0.77$ and $6.82 \pm 1.01 \mathrm{AU}$ compared to the NTC for the TC and TL respectively. In comparison, the reference products all delivered marginal changes in SCH that reached maximum increases of $3.65 \pm 1.21$ after $6 \mathrm{~h}, 2.21 \pm 0.98$ after $6 \mathrm{~h}$, and $1.75 \pm 1.09$ after $12 \mathrm{~h}$ compared to the NTC for RC1, RC2 and RC3 respectively.

Visual dryness at baseline displayed good correlation with SCH levels measured using the corneometer, $r=-0.6151$. A single point on the 5-point visual scale corresponded to a change in SCH of approximately 6.67 AU. All treatments significantly reduced the appearance of skin surface dryness compared to the NTC at 3,6 and $12 \mathrm{~h}$ following application by comparable levels (approximately 1 point). However, differences in performance became apparent after $24 \mathrm{~h}$ where only RC1, TC and TL were associated with significant differences. Skin dryness was $1.2 \pm 1.0$ on average at the NTC site $24 \mathrm{~h}$ post-application, whereas sites treated with the reference products displayed slightly reduced mean scores for visual dryness at $1.0 \pm 1.0,0.9 \pm 0.9,0.9 \pm 1.0$ for RC1, RC2 and RC3 respectively. Mean visual skin dryness at sites treated with the TC and TL was lower still at $0.8 \pm 0.8$ and $0.8 \pm 0.8$ respectively.

\section{DISCUSSION}

The TC and TL both delivered sustained clinically relevant increases in $\mathrm{SCH}$ that lasted more than $24 \mathrm{~h}$ following a single application. In contrast the three reference products delivered marginal changes which did not reach a clinically significant level. A change in $\mathrm{SCH}$ of 5 units corresponds to the difference between active and inactive $\mathrm{AD}$ at non-lesional sites [12]. A decrease of 7 units corresponds to the difference between mild and moderate $\mathrm{AD}$ or between normal and intrinsic AD skin [13, 14]. A greater difference of 13 units is observed between moderate and severe $\mathrm{AD}$ and between the skin of intrinsic and extrinsic AD patients. By increasing SCH by more than 6.5 units over a 24-h period following application, the TC and TL deliver clinically relevant levels of skin moisturization appropriate for the treatment of AD.

The capacitance data is supported by the visual dryness scores. Whilst the sensitivity of the visual dryness scale is notably reduced, and restricted to the superficial surface layers of the $\mathrm{SC}$ compared to the hydration measurements (a change in visual dryness corresponded to a change in 6.67 AU SCH here), the findings support a more efficacious and longer-lasting moisturization effect of the test products compared to two of the three reference products. RC1 was also associated with a significantly lower level of dryness $24 \mathrm{~h}$ following application, albeit to a smaller extent than the test products, but this was not supported by the $\mathrm{SCH}$ data. As a result of the low sensitivity of the visual dryness scores it was not possible to separate the effects of the treatments.

The findings of this study reflect those of a previous study comparing the effects of a single application of three other emollients; Aqueous cream (AC), Diprobase cream (DC) and Doublebase gel (DG) [12]. AC and DC are similar in composition to the reference products tested here. DG is distinct owing to the presence of the active moisturizing agent glycerol in a gel base. DG was significantly more hydrating than AC or DC; however, none of the products tested exhibited a sustained clinically meaningful 
(a)

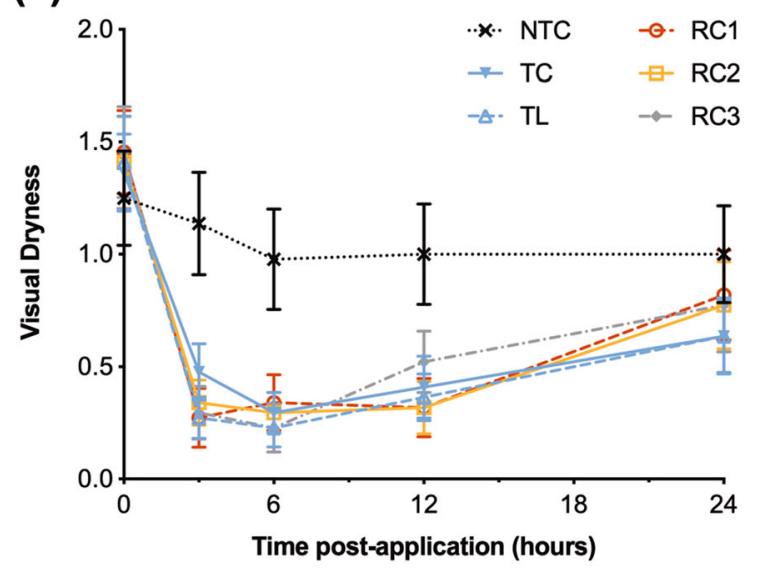

(c)

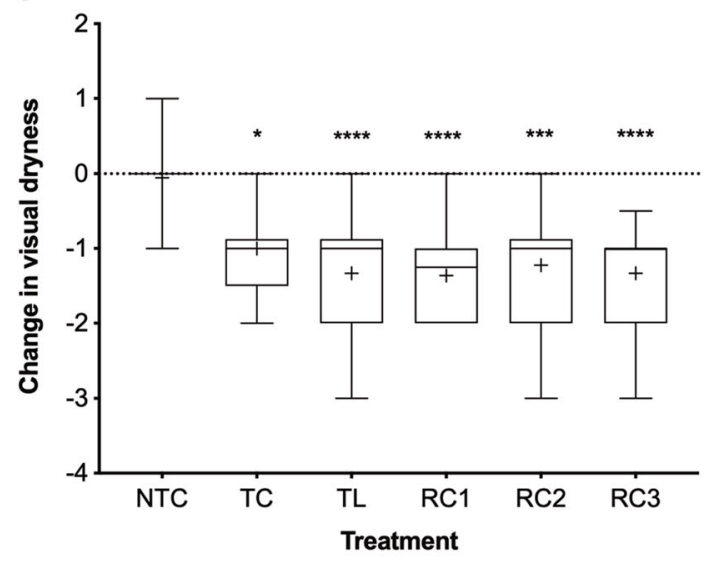

(e)

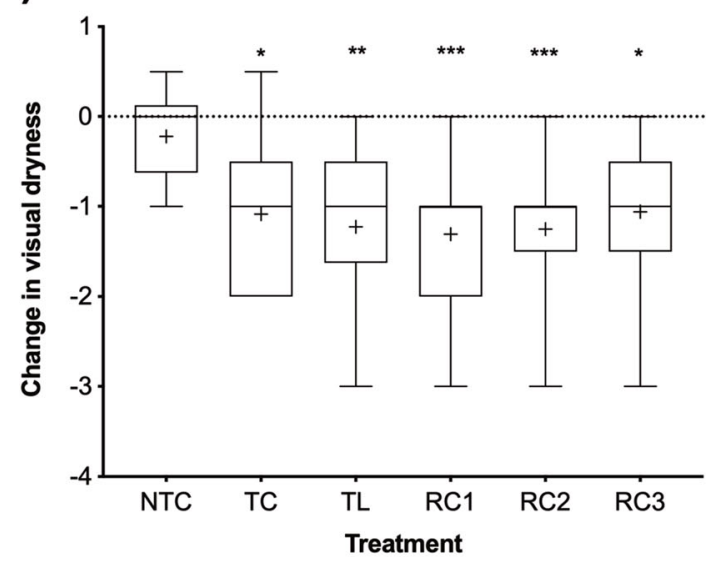

(b)

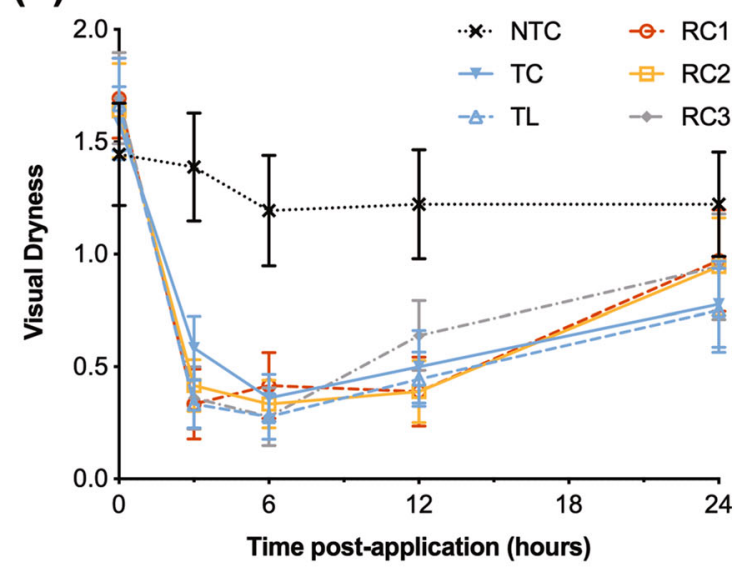

(d)

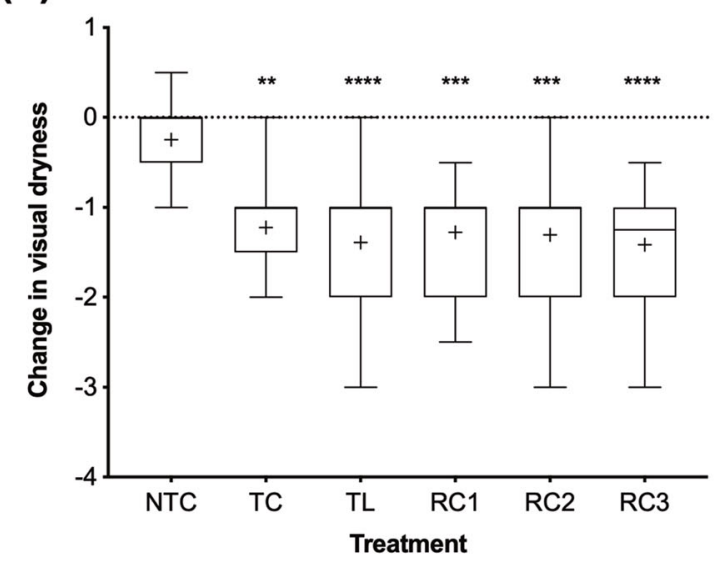

(f)

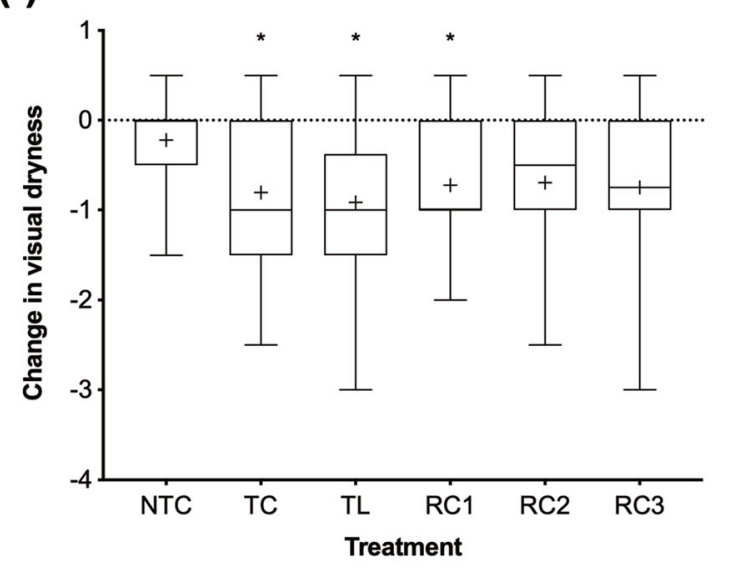


4Fig. 2 Effect of the 6 treatment conditions on visual dryness (secondary analysis). Raw visual dryness score over $24 \mathrm{~h}$ following a single application is displayed for the FAS (a) and PPS (b). Change in visual dryness from baseline is displayed for the PPS at $3 \mathrm{~h} \mathrm{(c),6} \mathrm{h} \mathrm{(d),} 12 \mathrm{~h}(\mathbf{e})$ and $24 \mathrm{~h}$ (f) post-treatment. Baseline measurements $(0 \mathrm{~h})$ were taken immediately prior to application of the investigational products. NTC no treatment control, TC test cream, TL test lotion, RC reference cream. Error bars (a and b) indicate SEM. Boxes (c-f) indicate the median, 25th and 75th percentiles, with ' + ' for the mean and whiskers indicating the minimum and maximum. Asterisks indicate the results of a Dunn's post-test comparing each treatment to the NTC $\quad\left({ }^{*} p<0.05,{ }^{* *} p<0.01\right.$, $\left.{ }^{* * *} p<0.001,{ }^{* * * *} p<0.0001\right)$

effect lasting $24 \mathrm{~h}$. There is moderate clinical evidence that glycerol-containing moisturizers, which impart greater increases in $\mathrm{SCH}$, are more clinically efficacious compared to non-glycerolcontaining moisturizers [15]. Whilst this demonstrates the link between $\mathrm{SCH}$ and clinical efficacy in treating $\mathrm{AD}$, it does not explain the longer-lasting effects of the test products here. In addition to glycerol the test products contain a range of skin lipids in an MVE vehicle. This combination of skin lipids and glycerol significantly increases moisturization potential above that of glycerol alone (by more than twofold) [16]. Moreover, MVE technology controls the release of active agents to prolong their therapeutic effect [17]. To this end we show here that the combination of glycerol and skin lipids in an MVE vehicle significantly increase and prolong SCH compared to traditional emollients without these ingredients.

The study is limited by its narrow focus on moisturization potential after a single application. Repeated applications are expected to impart additive effects. Skin dryness measurements are included to confirm capacitance measurements of $\mathrm{SCH}$. Such visual observations are limited by their relative insensitivity and the baseline level of dryness. The results can be readily generalized to male and female adults prone to $\mathrm{AD}$ with dry skin, and the effects of the reference products in this population agree with previously published reports $[11,12,17]$. The ethnic diversity of the population was low, but representative of the population engaging in research locally. Our intention is to follow this preliminary study with a vehicle-controlled trial investigating the effects of a treatment regimen with the TC on skin barrier structure and function, in a similar population stratified by age.

\section{CONCLUSIONS}

The findings of this study demonstrate the superior moisturization activity of the test products, and suggest a concordantly greater clinical efficacy [15]. Importantly at this stage, and consistent with our hypothesis, a sustained effect on both hydration and skin dryness lasting more than $24 \mathrm{~h}$ is reported for the test products, but not for any of the reference products. This means that the health of the skin can be improved with once-daily applications of the test products as opposed to the 2-4 applications per day recommended for traditional emollients [18]. As a result of the high volume of prescriptions for traditional emollients, this data suggests that many patients would benefit from improved skin moisturization and reduced therapeutic burden by switching to one of the test products.

\section{ACKNOWLEDGEMENTS}

We are very grateful to all of our volunteers who have given up their time to take part in this study; thank you.

Funding. This investigator-led study was funded by L'Oreal Active Cosmetics Division (UK) (154896), who is also funding the journals Rapid Service fee.

Authorship. All named authors meet the International Committee of Medical Journal Editors (ICMJE) criteria for authorship for this article, take responsibility for the integrity of the work as a whole, and have given their approval for this version to be published.

Disclosure of interest. Simon G. Danby has received research grants from, participated in 
advisory boards for, or has consulted with Almirall, Astellas Pharma, Bayer, Harvey Water Softeners, Hyphens Pharma, Leo Pharma, L'Oreal, Johnson \& Johnson, MSD, Perrigo, Pfizer, and Stiefel-GSK. Michael J. Cork is an investigator and consultant for Astellas, Almirall, Bayer, Boots, Galapagos, Hyphens, Johnson \& Johnson, Leo Pharma, L'Oreal, Menlo, MSD, Novartis, Oxagen, Pfizer, Procter \& Gamble, Perrigo, Regeneron Pharmaceuticals, Inc., and Sanofi Genzyme, Stiefel-GSK. Paul V. Andrew, Kirsty Brown, John Chittock and Linda J. Kay have nothing to disclose.

Compliance with Ethics Guidelines. The University of Sheffield Research Ethics Committee (UREC) approved the study, under the project reference 018584 . The study was performed in accordance with the Helsinki Declaration of 1964 , and its later amendments, and all subjects provided informed consent to participate.

Data Availability. The datasets generated during and/or analyzed during the current study are available from the corresponding author on reasonable request.

Open Access. This article is licensed under a Creative Commons Attribution-NonCommercial 4.0 International License, which permits any non-commercial use, sharing, adaptation, distribution and reproduction in any medium or format, as long as you give appropriate credit to the original author(s) and the source, provide a link to the Creative Commons licence, and indicate if changes were made. The images or other third party material in this article are included in the article's Creative Commons licence, unless indicated otherwise in a credit line to the material. If material is not included in the article's Creative Commons licence and your intended use is not permitted by statutory regulation or exceeds the permitted use, you will need to obtain permission directly from the copyright holder. To view a copy of this licence, visit http://creativecommons.org/licenses/by$\mathrm{nc} / 4.0 /$.

\section{REFERENCES}

1. Di Nardo A, Wertz P, Giannetti A, et al. Ceramide and cholesterol composition of the skin of patients with atopic dermatitis. Acta Derm Venereol. 1998;78(1):27-30.

2. Meguro S, Arai Y, Masukawa Y, et al. Relationship between covalently bound ceramides and transepidermal water loss (TEWL). Arch Dermatol Res. 2000;292(9):463-8.

3. Proksch E, Jensen JM, Elias PM. Skin lipids and epidermal differentiation in atopic dermatitis. Clin Dermatol. 2003;21(2):134-44.

4. Fluhr JW, Darlenski R, Taieb A, et al. Functional skin adaptation in infancy-almost complete but not fully competent. Exp Dermatol. 2010;19(6): 483-92.

5. Ghadially R, Brown BE, Sequeira-Martin SM, et al. The aged epidermal permeability barrier. Structural, functional, and lipid biochemical abnormalities in humans and a senescent murine model. J Clin Investig. 1995;95(5):2281-90.

6. Wilhelm KP, Cua AB, Maibach HI. Skin aging. Effect on transepidermal water loss, stratum corneum hydration, skin surface $\mathrm{pH}$, and casual sebum content. Arch Dermatol. 1991;127(12):1806-9.

7. Mischo M, von Kobyletzki LB, Bründermann E, et al. Similar appearance, different mechanisms: xerosis in HIV, atopic dermatitis and ageing. Exp Dermatol. 2014;23:446-8.

8. Elias PM. Lipid abnormalities and lipid-based repair strategies in atopic dermatitis. Biochim Biophys Acta. 2014;1841(3):323-30.

9. Zeichner JA, Del Rosso JQ. Multivesicular emulsion ceramide-containing moisturizers: an evaluation of their role in the management of common skin disorders. J Clin Aesthet Dermatol. 2016;9(12): 26-322.

10. Draelos ZD. The effect of ceramide-containing skin care products on eczema resolution duration. Cutis. 2008;81(1):87-91.

11. Danby SG, Chittock J, Brown K, et al. The effect of tacrolimus compared with betamethasone valerate on the skin barrier in volunteers with quiescent atopic dermatitis. $\mathrm{Br}$ J Dermatol. 2014;170(4): 914-21.

12. Danby SG, Chalmers J, Brown K, et al. A functional mechanistic study of the effect of emollients on the structure and function of the skin barrier. $\mathrm{Br} \mathrm{J}$ Dermatol. 2016;175(5):1011-9. 
13. Addor FA, Takaoka R, Rivitti EA, et al. Atopic dermatitis: correlation between non-damaged skin barrier function and disease activity. Int J Dermatol. 2012;51(6):672-6.

14. Choi SJ, Song MG, Sung WT, et al. Comparison of transepidermal water loss, capacitance and $\mathrm{pH}$ values in the skin between intrinsic and extrinsic atopic dermatitis patients. J Korean Med Sci. 2003;18(1):93-6.

15. van Zuuren EJ, Fedorowicz Z, Christensen R, et al. Emollients and moisturisers for eczema. Cochrane Database Syst Rev. 2017;2(2):CD012119.
16. Huang HC, Chang TM. Ceramide 1 and ceramide 3 act synergistically on skin hydration and the transepidermal water loss of sodium lauryl sulfateirritated skin. Int J Dermatol. 2008;47(8):812-9.

17. Bikowski J, Shroot B. Multivesicular emulsion: a novel, controlled-release delivery system for topical dermatological agents. J Drugs Dermatol. 2006;5(10):942-6.

18. PCDS, BAD. Primary Care Dermatology Society and British Association of Dermatologists Guidelines on the management of atopic eczema. Guidelines. 2009. https://www.nice.org.uk/guidance/ta81/resources/ primary-care-dermatology-society2. Accessed 14 July 2020. 